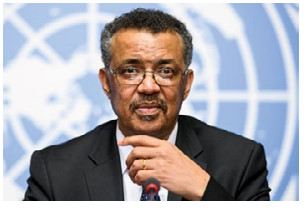

Credit: FABRICE COFFRINI / Staff/AFP/ Getty

\section{It takes knowledge to transform the world to be a better place to grow older}

\author{
In support of the Decade of Healthy Aging, the World Health Organization has launched a \\ publicly available, multi-lingual knowledge exchange platform that will enable people to find, \\ share and produce knowledge on healthy aging. The WHO Director-General introduces the \\ platform and explains how it can be used to make the world a better place to grow older.
}

$\mathrm{D}$ uring the next ten years, the number of people aged 60 years or over globally is projected to grow by 38 per cent, from 1 billion to 1.4 billion, outnumbering youth. This increase will be greatest and most rapid in low- and lowermiddle-income countries. Despite the predictability of population aging and its accelerating pace, the world is not prepared. The devastating impact of COVID-19 on older populations has shed light on the many gaps in our systems and services, such as the availability and quality of health and social care. It has also influenced how we think about aging itself, through the all too common-yet false-narrative that all older people are frail and vulnerable.

The United Nations General Assembly, recognising the need for collective and concerted multi-sectoral action, declared 2021-2030 the Decade of Healthy Aging. The World Health Organization was asked to lead a global collaboration to foster longer and healthier lives, bringing together governments, civil society, the private sector, media and United Nations agencies.

The plan for the Decade prioritises four action areas to improve the lives of older people, their families and their communities. These interlinked action areas include changing how we think, feel and act towards age and aging; fostering the abilities of older people within their communities; delivering integrated care and primary health services that are responsive to the needs of older people; and providing access to long-term care for older people who need it. These actions are supported by four enablers: voice and engagement of older people, families and communities; nurturing leadership and capacity building; connecting stakeholders; and improving data, research and innovation.

The promise and potential of the Decade of Healthy Aging can only be achieved if we work together across countries, sectors and disciplines. WHO, in collaboration with stakeholders from over 80 countries, has developed an innovative, multi-lingual knowledge exchange platform that will enable people to find, share and produce knowledge on healthy aging. The design of the Decade Platform (https://www. decadeofhealthyaging.org/) recognizes that there are many types and sources of knowledge, including those derived from personal experience. Some knowledge is explicit while other knowledge is tacit, such as that of long-term care providers who have identified ways to reduce anxiety in older people without necessarily being able to explain how they did it. To meet different knowledge needs, the platform contains user guides, reports, databases, and teaching and research materials, as well as informal and face-to-face learning opportunities.

The Decade Platform is designed for use by policy makers, practitioners and all other stakeholders to find, share and produce the knowledge needed for their work to foster healthy aging. Submissions to the Platform are open to the public, and all users can access and make available knowledge across five core areas corresponding to the Decade's enablers. Every knowledge item on the Platform is reviewed by a group of expert volunteers. For example, users can access a data portal that brings together data on global indicators for monitoring the health and well-being of older people, such as percentage of people aged 60 years and over, healthy life expectancy at age 60 , major causes of death in older people, percentage of older people receiving long-term care, and percentage of older people living in an age-friendly environment.

To maximize the use of knowledge, the platform also offers stakeholders training and opportunities to share successful initiatives and good practices. The programme " Healthy Aging for Impact in the 21st Century: Global Online Leaders Training" has already trained over 800 government officials, focal points for aging in relevant ministries, United Nations staff in country offices, and those working in civil society organizations in the field of aging.

There is also a need for new knowledge, both theoretical and practical. The Decade Platform provides an opportunity to make gaps visible and provide the tools and resources necessary to address them. This includes the documentation and dissemination of new knowledge gained not only through field reports, but also by amplifying the voices, stories and perspectives of older people, their families and their communities to find out what matters most.

The ultimate decisions about how to foster healthy aging rest with national governments and civil society. Being able to access the wealth of diverse knowledge in different languages and relevant to different disciplines and contexts can facilitate that process. Knowledge needs will evolve, and the platform will evolve in response. The Decade Platform works to bring together diverse knowledge to improve the lives of older people, their families and communities, now and in the future.

Transforming our world to improve the quality of life for aging populations is a moral, social and economic imperative for current and future generations. The Sustainable Development Goals provide the vision, the UN Decade of Healthy Aging the framework and the Decade Platform the vehicle for ensuring that none of us are left behind as we age.

\section{Tedros Adhanom Ghebreyesus ${ }^{\square}$ World Health Organization, Geneva, Switzerland.

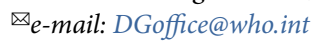

Published online: 8 September 2021 https://doi.org/10.1038/s43587-021-00120-9

Competing interests

The author declares no competing interests. 